\title{
Upfront radiation versus EGFR-TKI: which is the best approach for EGFR-mutated NSCLC patients with brain metastasis?
}

\author{
Francesco Passiglia ${ }^{1,2}$, Christian Rolfo ${ }^{2}$ \\ ${ }^{1}$ Department of Surgical, Oncological and Oral Sciences, Section of Medical Oncology, University of Palermo, Palermo, Italy; ${ }^{2} \mathrm{Phase}$ I - Early \\ Clinical Trials Unit, Oncology Department, Antwerp University Hospital and Center for Oncological Research (CORE), Antwerp University, \\ Antwerp, Belgium \\ Correspondence to: Prof. Dr. Christian Rolfo, MD, PhD, MBAh, Head of Phase I - Early Clinical Trials Unit, Director of Clinical Trial Management \\ Program. Oncology Department, University Hospital Antwerp, UZA, Wilrijkstraat 10, 2650 Edegem, Belgium. Email: Christian.Rolfo@uza.be. \\ Comment on: Magnuson WJ, Lester-Coll NH, Wu AJ, et al. Management of Brain Metastases in Tyrosine Kinase Inhibitor-Naïve Epidermal Growth \\ Factor Receptor-Mutant Non-Small-Cell Lung Cancer: A Retrospective Multi-Institutional Analysis. J Clin Oncol 2017;35:1070-1077.
}

Submitted Mar 27, 2017. Accepted for publication Mar 31, 2017.

doi: $10.21037 /$ tcr.2017.04.08

View this article at: http://dx.doi.org/10.21037/tcr.2017.04.08

In The Fournal of Clinical Oncology, William J. Magnuson (1) and colleagues have recently reported the results of a multicenter retrospective analysis comparing the impact of three different treatment strategies on survival outcomes of 351 patients with epidermal growth factor receptor (EGFR) mutated non-small cell lung cancer (NSCLC) and brain metastases (BM). Treatment options included stereotactic radiosurgery (SRS) followed by EGFR-TKI $(n=100)$, whole-brain radiotherapy (WBRT) followed by EGFR-TKI $(n=120)$, or EGFR-TKI followed by SRS or WBRT at the time of intracranial progression $(n=131)$. Results showed a significantly longer median overall survival (OS) in patients who received upfront SRS (46 months) as compared to WBRT (30 months) or upfront TKI (25 months) $(\mathrm{P}<0.001)$, suggesting SRS followed by EGFR-TKI as the best treatment approach for these patients. The significant survival improvement was independent from significant prognostic covariables such as age, ECOG performance status, number of BM, EGFR mutation, and extracranial metastases, which were included in the multivariable analysis. The upfront use of radiotherapy resulted also in a significantly longer median time to intracranial progression (23 vs. 18 months; $\mathrm{P}=0.025)$ and 2 -year OS rate $(78 \%$ vs. $51 \% ; \mathrm{P}<0.001)$, and it was maintained regardless of patients' prognosis.

The results of several randomized phase III (2-9) studies convincingly and consistently demonstrated a significant superiority of upfront EGFR-TKI over platinum-based chemotherapy for the subgroup of patients whose tumors harbor an EGFR activating mutation, leading to a paradigm shift in the first-line treatment of these patients, to whom the current gold standard is starting with an EGFR-TKI, gefitinib, erlotinib, or afatinib. However, several years later the approval of the first EGFR-TKI in the clinical setting the specific role of these agents in the therapeutic strategy of patients with BM remains still debated.

Brain metastasis are a common event in EGFR-mutated NSCLC, with major negative impact on patients' quality of life (QoL) and survival, ranging from 4.5 to 11.0 months after the diagnosis $(10,11)$. Despite the recent innovations in the treatments of EGFR positive NSCLC, current options available for EGFR-mutated patients with BM are very limited, also because of their historical low accrual in prospective clinical trials. Surgical resection, SRS or WBRT concomitantly or followed by EGFR-TKIs remain the most common approaches to treat these patients (12). However the optimal treatment sequences and combinations have not been clarified yet. Activity of upfront firstgeneration EGFR-TKIs has been reported in retrospective series including low number of East-Asian patients (13-16), overall showing encouraging intracranial response rates (RR) despite to the low penetration of such agents across the blood-brain barrier (BBB) $(17,18)$. Some studies showed that erlotinib has a better central nervous system (CNS) penetration than gefitinib (19), thus suggesting it as preferred option for asymptomatic patients with BM. 
Interestingly the second-generation EGFR-TKI afatinib has shown encouraging activity in NSCLC patients with BM (20). Gerber et al. retrospectively analyzed the survival outcomes of 222 patients with EGFR-positive NSCLC and newly diagnosed $\mathrm{BM}$, showing no OS differences between WBRT and erlotinib, but a significant longer intracranial control in favour of the WBRT group (15). These data have been confirmed by a recent meta-analysis including 12 non-comparative trials and more than 300 EGFR-mutated NSCLC patients with BM revealing that WBRT improved both intracranial control and survival outcomes as compared to upfront EGFR-TKI monotherapy (21). Even if limited by methodological limitations, these studies underscored the important role of upfront WBRT in the management of BM, but didn't provide any evidences about SRS efficacy because of the very low number of patients treated with SRS in the included trials. In this scenario the study by Magnuson et al. (1) offered the opportunity to clarify a controversial and actual question: may radiotherapy be replaced by or deferred after upfront EGFR-TKI in patients with EGFRpositive NSCLC and BM? The results of this trial clearly demonstrated that deferring brain radiotherapy after EGFRTKI led to inferior survival in the analyzed population, suggesting SRS followed by EGFR-TKI as the optimal treatment sequence for this subgroup of patients, regardless from significant prognostic factors. Furthermore the study addressed the controversy over the use of WBRT. Indeed the 120 patients who received WBRT followed by TKI reported a median survival of 30 months, which was significantly lower than the SRS-TKI sequence (45 months), even if they were more likely to have a less favorable prognosis. Since QoL is a relevant outcome for patients with metastatic disease, the use of SRS could allow patients to avoid the potential acute and late neurological toxicities associated with WBRT, often responsible for the patients' clinical decline. Unfortunately Magnuson $e t$ al. didn't collect adverse events (AEs), thus lacking a great opportunity to analyze both safety and QoL related to the different treatment strategies in a large real word population. Furthermore the results of this study should be cautiously interpreted because of the inherent methodological limitations. First the retrospective design of the study, even if partially corrected by the application of the propensity score analysis, may expose to significant selection biases, ultimately affecting the final results. Indeed the low percentage of patients with extracranial disease as well as the lower percentage of patients with stage IV assigned to the SRS arm, as compared to both WBRT and EGFR-TKI arms, could have influenced the survival analysis in favour of upfront SRS. Furthermore the exclusion of patients who didn't receive EGFR-TKI after brain radiation or radiotherapy after TKI-failure could also have affected the survival analysis. Finally it's interesting to observe how medical oncologists considered upfront EGFR-TKI an effecting option for patients with asymptomatic disease, while WBRT was administered in patients with more than 5-10 BM who showed the strongest trend toward inferior OS, and SRS was preferred in patients with oligometastatic symptomatic disease. To summarize the study by Magnuson et al. (1) provide an interesting contribution to the current scientific debate supporting SRS as upfront treatment in the management of EGFRpositive NSCLC patients with BM. However, as declared by the authors, prospective randomized trials comparing these different strategies are urgently needed in order to definitively identify the optimal treatment approach for this subgroup of NSCLC patients. Recently the results of the first prospective randomized trial comparing EGFRTKI icotinib $v s$. WBRT \pm chemotherapy in EGFR-mutated Asian NSCLC patients with BM, have shown a significant superiority of TKI in terms of both intracranial and systemic $\mathrm{RR}$, as well as intracranial and systemic progression free survival (PFS), together with a lower incidence of severe treatment-related AEs (22). These data suggest icotinib as new standard 1-line treatment for EGFR-positive Asian patients with BM. However data on OS, neurotoxicity and secondary use of WBRT are not available yet. Furthermore it will be useful to see the results of other two ongoing randomized phase III trials comparing upfront EGFRTKI erlotinib or gefitinib vs. WBRT (NCT02714010; NCT02338011) including also Caucasian population before to modify current treatment recommendations in the setting of patients. Besides these, randomized studies of SRS followed by EGFR-TKI vs. EGFR-TKI followed by SRS would be even more useful to clarify the optimal treatment sequence in this population. Concurrent administration of brain radiotherapy and EGFR-TKI could represent another promising approach allowing to combine the intracranial and the systemic control obtained with SRS and TKI, respectively. Pre-clinical data showed a synergistic effect between EGFR-inhibition and radiotherapy (23), likely due to the radio-sensitizing effect of TKIs and to the damage of BBB caused by radiation. Different phase II studies demonstrated a tolerable safety profile and encouraging activity of WBRT and EGFR-TKI combination in EGFRpositive NSCLC patients with BM $(24,25)$. The prospective randomized TRACTS study (NCT01763385) is currently 
investigating WBRT plus erlotinib combination $v s$. erlotinib alone in this subset of patients, and results are eagerly awaited. As regards new treatment options, encouraging data emerged from the phase I BLOOM study including EGFRmutated NSCLC patients progressed on prior treatment with EGFR-TKI with a confirmed diagnosis of BM. Among the 21 patients receiving osimertinib $160 \mathrm{mg}$ daily, $33 \%$ had partial response (PR) and $43 \%$ had stable disease (SD), with a tolerable safety profile including only $14 \%$ grade $\geq 3$ drugrelated AEs (26). Waiting for the results in the T790Mpositive cohort, osimertinib has already shown high activity in patients with CNS disease harboring T790M mutation enrolled in two phase II studies (26), likely due to its greater penetration of animal models' BBB compared to other TKIs, gefitinib, rociletinib, or afatinib (27). Furthermore the recent results of the AURA3 trial demonstrated a significant survival benefit of osimertinib over platinum-chemotherapy in 144 T790M-positive patients with CNS metastasis at baseline, suggesting it as a very effective option in this population (28).

In conclusion the study of Magnuson $\mathrm{et} \mathrm{al}$. represents a significant attempt to improve the management of EGFRmutated NSCLC patients with BM. However, as mentioned before, such results should be interpreted taking into account the new treatment options such as third-generation TKI which will be early available for these patients. Finally, the results of prospective ongoing randomized trials will be crucial to define the optimal treatment approach for each patient with EGFR-positive NSCLC and BM.

\section{Acknowledgments}

Funding: None.

\section{Footnote}

Provenance and Peer Review: This article was commissioned and reviewed by the Section Editor Shaohua Cui (Department of Pulmonary Medicine, Shanghai Chest Hospital, Shanghai Jiao Tong University, Shanghai, China).

Conflicts of Interest: Both authors have completed the ICMJE uniform disclosure form (available at http://dx.doi. org/10.21037/tcr.2017.04.08). The authors have no conflicts of interest to declare.

Ethical Statement: The authors are accountable for all aspects of the work in ensuring that questions related to the accuracy or integrity of any part of the work are appropriately investigated and resolved.

Open Access Statement: This is an Open Access article distributed in accordance with the Creative Commons Attribution-NonCommercial-NoDerivs 4.0 International License (CC BY-NC-ND 4.0), which permits the noncommercial replication and distribution of the article with the strict proviso that no changes or edits are made and the original work is properly cited (including links to both the formal publication through the relevant DOI and the license). See: https://creativecommons.org/licenses/by-nc-nd/4.0/.

\section{References}

1. Magnuson WJ, Lester-Coll NH, Wu AJ, et al. Management of Brain Metastases in Tyrosine Kinase Inhibitor-Naïve Epidermal Growth Factor ReceptorMutant Non-Small-Cell Lung Cancer: A Retrospective Multi-Institutional Analysis. J Clin Oncol 2017;35:1070-7.

2. Mitsudomi T, Morita S, Yatabe $Y$, et al. Gefitinib versus cisplatin plus docetaxel in patients with non-small-cell lung cancer harbouring mutations of the epidermal growth factor receptor (WJTOG3405): an open label, randomised phase 3 trial. Lancet Oncol 2010;11:121-8.

3. Maemondo M, Inoue A, Kobayashi K, et al. Gefitinib or chemotherapy for non-small-cell lung cancer with mutated EGFR. N Engl J Med 2010;362:2380-8.

4. Mok TS, Wu YL, Thongprasert S, et al. Gefitinib or carboplatin-paclitaxel in pulmonary adenocarcinoma. $\mathrm{N}$ Engl J Med 2009;361:947-57.

5. Rosell R, Carcereny E, Gervais R, et al. Erlotinib versus standard chemotherapy as first-line treatment for European patients with advanced EGFR mutation-positive non-small-cell lung cancer (EURTAC): a multicentre, open-label, randomised phase 3 trial. Lancet Oncol 2012;13:239-46.

6. Zhou C, Wu YL, Chen G, et al. Erlotinib versus chemotherapy as first-line treatment for patients with advanced EGFR mutation-positive non-small-cell lung cancer (OPTIMAL, CTONG-0802): a multicentre, open-label, randomised, phase 3 study. Lancet Oncol 2011;12:735-42.

7. Sequist LV, Yang JC, Yamamoto N, et al. Phase III study of afatinib or cisplatin plus pemetrexed in patients with metastatic lung adenocarcinoma with EGFR mutations. J Clin Oncol 2013;31:3327-34.

8. Wu YL, Zhou C, Hu CP, et al. Afatinib versus cisplatin 
plus gemcitabine for first-line treatment of Asian patients with advanced non-small-cell lung cancer harbouring EGFR mutations (LUX-Lung 6): an open-label, randomised phase 3 trial. Lancet Oncol 2014;15:213-22.

9. Yang JC, Wu YL, Schuler M, et al. Afatinib versus cisplatin-based chemotherapy for EGFR mutation-positive lung adenocarcinoma (LUX-Lung 3 and LUX-Lung 6): analysis of overall survival data from two randomised, phase 3 trials. Lancet Oncol 2015;16:141-51.

10. Liao BC, Lee JH, Lin CC, et al. Epidermal Growth Factor Receptor Tyrosine Kinase Inhibitors for NonSmall-Cell Lung Cancer Patients with Leptomeningeal Carcinomatosis. J Thorac Oncol 2015;10:1754-61.

11. Umemura S, Tsubouchi K, Yoshioka H, et al. Clinical outcome in patients with leptomeningeal metastasis from non-small cell lung cancer: Okayama Lung Cancer Study Group. Lung Cancer 2012;77:134-9.

12. Novello S, Barlesi F, Califano R, et al. Metastatic nonsmall-cell lung cancer: ESMO Clinical Practice Guidelines for diagnosis, treatment and follow-up. Ann Oncol 2016;27:v1-v27.

13. Park SJ, Kim HT, Lee DH, et al. Efficacy of epidermal growth factor receptor tyrosine kinase inhibitors for brain metastasis in non-small cell lung cancer patients harboring either exon 19 or 21 mutation. Lung Cancer 2012;77:556-60.

14. Iuchi T, Shingyoji M, Sakaida T, et al. Phase II trial of gefitinib alone without radiation therapy for Japanese patients with brain metastases from EGFR-mutant lung adenocarcinoma. Lung Cancer 2013;82:282-7.

15. Gerber NK, Yamada Y, Rimner A, et al. Erlotinib versus radiation therapy for brain metastases in patients with EGFR-mutant lung adenocarcinoma. Int J Radiat Oncol Biol Phys 2014;89:322-9.

16. Zhang Q, Zhang X, Yan H, et al. Effects of epidermal growth factor receptor-tyrosine kinase inhibitors alone on EGFR-mutant non-small cell lung cancer with brain metastasis. Thorac Cancer 2016;7:648-54.

17. Zhao J, Chen M, Zhong W, et al. Cerebrospinal fluid concentrations of gefitinib in patients with lung adenocarcinoma. Clin Lung Cancer 2013;14:188-93.

18. de Vries NA, Buckle T, Zhao J, et al. Restricted brain penetration of the tyrosine kinase inhibitor erlotinib due to the drug transporters P-gp and BCRP. Invest New Drugs 2012;30:443-9.

19. Togashi Y, Masago K, Masuda S, et al. Cerebrospinal fluid concentration of gefitinib and erlotinib in patients with non-small cell lung cancer. Cancer Chemother Pharmacol
2012;70:399-405.

20. Hoffknecht P, Tufman A, Wehler T, et al. Efficacy of the irreversible ErbB family blocker afatinib in epidermal growth factor receptor (EGFR) tyrosine kinase inhibitor (TKI)-pretreated non-small-cell lung cancer patients with brain metastases or leptomeningeal disease. J Thorac Oncol 2015;10:156-63.

21. Soon YY, Leong CN, Koh WY, et al. EGFR tyrosine kinase inhibitors versus cranial radiation therapy for EGFR mutant non-small cell lung cancer with brain metastases: a systematic review and meta-analysis. Radiother Oncol 2015;114:167-72.

22. Wu YL, Yang JJ, Zhou C, et al. BRAIN: A Phase III Trial Comparing WBI and Chemotherapy with Icotinib in NSCLC with Brain Metastases Harboring EGFR Mutations (CTONG 1201). J Thorac Oncol 2017;12:S6.

23. Chinnaiyan P, Huang S, Vallabhaneni G, et al. Mechanisms of enhanced radiation response following epidermal growth factor receptor signaling inhibition by erlotinib (Tarceva). Cancer Res 2005;65:3328-35.

24. Welsh JW, Komaki R, Amini A, et al. Phase II trial of erlotinib plus concurrent whole-brain radiation therapy for patients with brain metastases from non-small-cell lung cancer. J Clin Oncol 2013;31:895-902.

25. Ma S, Xu Y, Deng Q, et al. Treatment of brain metastasis from non-small cell lung cancer with whole brain radiotherapy and Gefitinib in a Chinese population. Lung Cancer 2009;65:198-203.

26. Yang JC, Kim DW, Kim SW, et al. Osimertinib activity in patients (pts) with leptomeningeal (LM) disease from non-small cell lung cancer (NSCLC): Updated results from BLOOM, a phase I study. J Clin Oncol 2016;34 suppl:abstr 9002.

27. Ballard P, Yates JW, Yang Z, et al. Preclinical Comparison of Osimertinib with Other EGFR-TKIs in EGFR-Mutant NSCLC Brain Metastases Models, and Early Evidence of Clinical Brain Metastases Activity. Clin Cancer Res 2016;22:5130-40.

28. Mok TS, Wu YL, Ahn MJ, et al. Osimertinib or PlatinumPemetrexed in EGFR T790M-Positive Lung Cancer. N Engl J Med 2017;376:629-40.

Cite this article as: Passiglia F, Rolfo C. Upfront radiation versus EGFR-TKI: which is the best approach for EGFRmutated NSCLC patients with brain metastasis? Transl Cancer Res 2017;6(Suppl 3):S533-S536. doi: 10.21037/tcr.2017.04.08 